Proceedings

\title{
Designing Ultra-Small Nanostructured Lipid Carriers: Critical Process Parameters ${ }^{\dagger}$
}

\author{
Maria Mendes 1,2,3, João Basso 1,2, João Sousa ${ }^{1,2}$, Alberto Pais ${ }^{2}$ and Carla Vitorino 1,2,3,* \\ 1 Faculty of Pharmacy, University of Coimbra, Pólo das Ciências da Saúde, Azinhaga de Santa Comba, \\ 3000-548 Coimbra, Portugal; mariamendes1093@gmail.com (M.M.); joaobasso@ff.uc.pt (J.B.); \\ jjsousa@ff.uc.pt (J.S.) \\ 2 Coimbra Chemistry Centre, Department of Chemistry, University of Coimbra, Rua Larga, \\ 3004-535 Coimbra, Portugal; pais@qui.uc.pt \\ 3 Centre for Neurosciences and Cell Biology (CNC), Faculty of Medicine, University of Coimbra, Rua Larga, \\ Pólo I, 1st Floor, 3004-504 Coimbra, Portugal \\ * Correspondence: csvitorino@ff.uc.pt; Tel.: +351-239-487-388 \\ + Presented at the 1st International Electronic Conference on Pharmaceutics, 1-15 December 2020; \\ Available online: https://iecp2020.sciforum.net/.
}

Citation: Mendes, M.; Basso, J.; Sousa, J.; Pais, A.; Vitorino, C. Designing Ultra-Small Nanostructured Lipid Carriers: Critical Process Parameters. Proceedings 2021, 78, 50. https:// doi.org/10.3390/IECP2020-08691

Published: 1 December 2020

Publisher's Note: MDPI stays neutral with regard to jurisdictional claims in published maps and institutional affiliations.

Copyright: (C) 2020 by the authors. Licensee MDPI, Basel, Switzerland. This article is an open access article distributed under the terms and conditions of the Creative Commons Attribution (CC BY) license (http://creativecommons.org/licenses /by/4.0/).

\begin{abstract}
Nanoparticles (NPs) offer noteworthy advantages in the treatment of several diseases by prompting, among other benefits, the site-specific delivery of drugs. Ultra-small nanostructured lipid carriers (usNLCs) are no exception. These correspond to a class of NPs composed of a blend of solid and liquid lipids, the latter usually in a higher proportion, which promotes a less ordered solid lipid matrix, providing a higher drug loading capacity, drug release modulation, and improved stability in comparison with other lipid nanoparticles. Several manufacturing methods have been described for obtaining usNLCs. However, a comprehensive understanding of the process is imperative to warrant the final quality of the NPs. In the present work, the hot high pressure homogenization ( $\mathrm{HPH}$ ) method, which is characterized by easy scaling-up, simplicity and ease of use, was used for the development of highly concentrated small size NLCs. Critical process parameters (CPPs) and critical material attributes (CMAs) were evaluated to investigate the manufacturing process reproducibility, inter-batch consistency, long-term formulation stability, drug loading capacity and drug release. To gain a broader understanding of this method, multivariate analysis was applied to investigate how the physicochemical properties of the usNLC are affected by the variation in CPPs/CMAs. CPPs include HPH-time, and HPH-pressure, while CMAs, such as lipid content, are also taken into consideration. The results show that a high lipid content $(15 \% w / w)$, an intermediate pressure and a short HPH time seem to be the crucial parameters to obtain both a small particle size $(<100 \mathrm{~nm})$ and a narrow size distribution (polydispersity index $<0.2)$ in usNLC prepared by the hot-HPH method, without affecting zeta potential $(>|30| \mathrm{mV})$.
\end{abstract}

Keywords: nanoparticles; lipid nanoparticles; critical process parameters; critical material attributes; ultra-small nanostructured lipid carriers

\section{Introduction}

Pharmaceutical development is an intensive and complex process. A good understanding of all stages is crucial to ensure the required quality of the final product. Therefore, following the principles of Quality by Design (QbD) facilitate the understanding of the relationship between material attributes and process parameters [1-3]. QbD is a systematic approach to development, based on prior knowledge and the quality risk management of the formulation components and the production method, ensuring the final product quality. In this way, QbD has been applied to the development of nanoparticles (NPs) with the aim of simplifying the manufacturing process and saving 
costs by implementing the quality specifications of the final product, as part of an overall control strategy.

Ultra-small nanostructured lipid carriers (usNLCs) exhibit particular colloidal properties that make them excellent candidates for drug delivery. In fact, they offer several advantages, including (i) the use of physiological, biocompatible and biodegradable lipids; (ii) higher encapsulation efficiency, drug loading and stability; (iii) controlled drug release; (iv) incorporation of lipophilic and hydrophilic molecules [4]. Several methods (temperature-or organic solvent-based) have been described in the literature for the production of usNLCs, including the hot or cold high-pressure homogenization (HPH), melt dispersion, solvent emulsification-evaporation, hydrophobic ion paring, double emulsion, among others. However, only the HPH is established for large-scale production, with additional cost-effective advantages [5-8].

Understanding the relationship between the critical process parameters (CPPs) and critical material attributes (CMAs) and the respective impact in the usNLC critical quality attributes (CQAs) is critical for performance improvement. In particular, the correlation between the HPH processing time and pressure (as CPPs), and the lipid content (as CMA) are herein addressed. The influence of these variables on physicochemical parameters, such as particle size, polydispersity index and zeta potential was analyzed to obtain usNLCs with quality in terms of efficiency and safety for cancer therapy.

\section{Experiments Materials}

Polysorbate 80 (Tween $^{\circledR} 80$ ) was provided by Sigma. Capryol ${ }^{\mathrm{TM}}$ PGMC (propylene glycol monocaprylate-type I) and Precirol ${ }^{\circledR}$ ATO 5 were kindly offered by Gattefossé (Gennevilliers, France). Lipoid S $75^{\circledR}$ (soy phospholipid) was provided by Lipoid GmbH (Ludwigshafen am Rhein, Germany). Ultrapure water (HPLC grade, 18.2 M $\Omega$ was prepared by means of a Milli-Q water apparatus (Millipore ${ }^{\circledR}$, Milford, MA, USA) and filtered through a $0.22 \mu \mathrm{m}$ nylon filter before use.

\subsection{Optimization of the Production Method of NLC}

The usNLCs were produced by hot high-pressure homogenization (hot-HPH). Modifications in lipid content, pressure, and processing time in hot HPH were evaluated to obtain the optimal conditions, according to a $3^{3}$ full factorial design (see Table 1). Briefly, the lipid phase consisting of a $(25: 75, \% w / w)$ mixture of solid (Precirol ${ }^{\circledR}$ ATO 5$)$ and liquid lipid (Capryol ${ }^{\mathrm{TM}}$ PGMC) and surfactant $\left(1 \% w / w\right.$, Lipoid ${ }^{\circledR}$ S75), was prepared and heated to $65{ }^{\circ} \mathrm{C}$. In parallel, the aqueous surfactant phase containing Tween ${ }^{\circledast} 80(5 \% \mathrm{w} / \mathrm{w})$ was prepared and heated up to $65^{\circ} \mathrm{C}$ before addition to the lipid phase. The mixture was then homogenized using a Ultra-Turrax X 10/25 (Ystral GmBh, Dottingen, Germany) at 24,000 rpm for $1 \mathrm{~min}$. The pre-emulsion formed was further processed using HPH (Emulsiflex C-3, Avestin, Mannheim, Germany) and the resulting dispersion was immediately cooled to $4{ }^{\circ} \mathrm{C}$. All samples were produced, at least, in triplicate.

Physicochemical characterization of usNLCs was performed in terms of particle size (PS), particle distribution (PI) and zeta potential (ZP) by dynamic and eletrophoretic light scattering, using a Zetasizer Nano ZS (Malvern Instruments, Malvern, UK) at $25{ }^{\circ} \mathrm{C}$. usNLC formulations were diluted with ultrapurified water (1:100) to generate an appropriate scattering intensity. 
Table 1. Design layout of different formulations.

\begin{tabular}{cccc}
\hline Independent Variables & \multicolumn{3}{c}{ Levels } \\
\cline { 2 - 4 } & $\mathbf{- 1}$ & $\mathbf{0}$ & $\mathbf{+ 1}$ \\
\hline Lipid content (\% w/w) & 10 & 12.5 & 15 \\
$\begin{array}{c}\text { High-pressure } \\
\text { homogenization (HPH) } \\
\text { time (min) }\end{array}$ & 2.5 & 5 & 7.5 \\
$\begin{array}{c}\text { High-pressure homoge- } \\
\text { nization (HPH) pressure } \\
(\text { bar) }\end{array}$ & 500 & 1000 & 1500 \\
\hline & \multicolumn{3}{c}{ Particle size (PS) } \\
\hline Dependent variables & \multicolumn{3}{c}{ Zeta potential (ZP) } \\
\cline { 2 - 4 } & & \\
\hline
\end{tabular}

\subsection{Multivariate Analyses}

A multivariate analysis is herein applied to inspect how the physicochemical properties of the usNLCs were influenced by the variation in CPPs and CMAs. Hierarchical cluster analysis (HCA) and principal component analysis (PCA) were performed resorting to JMP Pro 15.0.0 (SAS Institute Inc., Cary, NC, USA). Both methods require a spatial description of the usNLC formulations as points in Euclidean space. HCA and PCA are tools to explore hidden patterns, similarities, and differences among samples, where relationships within the data are not readily visible. HCA and PCA were applied after standard normalization to evaluate the quantitative and qualitative effects of CPPs and CMAs on PS, PI, and ZP. HCA and PCA were performed on the data set comprising 27 different formulations. A total of 3 defined predictors were considered corresponding to the lipid content of the formulation and the processing time and pressure in the HPH during the preparation of usNLCs. For HCA, the distance between clusters was calculated using Ward's minimum variance method, while PCA models were determined using the Rowwise estimation method and the correlation matrix.

\section{Results}

Optimization and Production of Unloaded usNLCs

Identifying the critical parameters, in terms of composition and processing, and inspecting how they affect the final dispersion is a major step in the development of nanocarriers. Multivariate analysis is a practical approach to evaluate the influence of the independent variables (CPPs and CMAs, HPH pressure and time, and lipid content, respectively) on the dependent variables (CQAs). These CQAs, including PS, PI and ZP, ultimately determine the physicochemical properties of usNLCs. In fact, these properties may influence their drug loading capacity, drug release behavior, stability in aqueous and biological media, biocorona formation tendency as well as other in vitro/in vivo parameters. Selecting the most suitable components and their concentrations requires a careful planning and optimization to achieve the desired outcome. The ideal PS, PI, and ZP of usNLCs were observed to be as low as possible PS and PI, and higher than $|30| \mathrm{mV}$ for zeta potential, along with the highest lipid content (Table 2). The composition trends can be monitored by combining (i) HCA exploiting the hierarchical distribution (Figure 1); (ii) PCA, with a biplot representation of the first two principal components (Figure 2); and (iii) a full factorial design, represented by contour plots (Figure 3). 
Table 2. Three-level, three-variable, $3^{3}$, full factorial design for the optimization of the production method of ultra-small nanostructured lipid carriers (usNLCs). The results are expressed as mean \pm standard deviation (SD). Formulations are highlighted in color, according to the clusters defined by hierarchical cluster analysis (HCA). Key: F: formulation; LC: lipid content; HPH: high-pressure homogenization; PS: particle size; ZP: zeta potential; PI: polydispersity index.

\begin{tabular}{ccccccc}
\hline $\mathbf{F}$ & $\mathbf{L C} \mathbf{( \% )}$ & HPH Time (min) & HPH Pressure (bar) & PS & PI & ZP \\
\hline $\mathbf{1}$ & 10 & 2.5 & 500 & $201 \pm 0.4$ & 0.414 & $-34.3 \pm 0.4$ \\
\hline $\mathbf{2}$ & 10 & 2.5 & 1000 & $112 \pm 0.5$ & 0.253 & $-36.0 \pm 0.5$ \\
\hline $\mathbf{3}$ & 10 & 2.5 & 1500 & $113 \pm 0.4$ & 0.254 & $-36.0 \pm 0.4$ \\
$\mathbf{4}$ & 10 & 5 & 500 & $153 \pm 1$ & 0.161 & $-43 \pm 1$ \\
\hline $\mathbf{5}$ & 10 & 5 & 1000 & $110 \pm 1$ & 0.245 & $-38 \pm 1$ \\
\hline $\mathbf{6}$ & 10 & 5 & 1500 & $171 \pm 2$ & 0.333 & $-35 \pm 2$ \\
\hline $\mathbf{7}$ & 10 & 7.5 & 500 & $120 \pm 1$ & 0.160 & $-29 \pm 1$ \\
\hline $\mathbf{8}$ & 10 & 7.5 & 1000 & $106 \pm 1$ & 0.205 & $-34 \pm 1$ \\
\hline $\mathbf{9}$ & 10 & 7.5 & 1500 & $157 \pm 1$ & 0.386 & $-35 \pm 1$ \\
\hline $\mathbf{1 0}$ & 12.5 & 2.5 & 500 & $116 \pm 2$ & 0.256 & $-38 \pm 2$ \\
\hline $\mathbf{1 1}$ & 12.5 & 2.5 & 1000 & $119 \pm 1$ & 0.260 & $-35 \pm 1$ \\
\hline $\mathbf{1 2}$ & 12.5 & 2.5 & 1500 & $115.7 \pm 0.4$ & 0.260 & $-32.0 \pm 0.4$ \\
\hline $\mathbf{1 3}$ & 12.5 & 5 & 500 & $137 \pm 1$ & 0.266 & $-37 \pm 1$ \\
\hline $\mathbf{1 4}$ & 12.5 & 5 & 1000 & $97 \pm 2$ & 0.246 & $-37 \pm 2$ \\
\hline $\mathbf{1 5}$ & 12.5 & 5 & 1500 & $100 \pm 1$ & 0.247 & $-35 \pm 2$ \\
\hline $\mathbf{1 6}$ & 12.5 & 7.5 & 500 & $100.5 \pm 0.6$ & 0.251 & $-37 \pm 1$ \\
\hline $\mathbf{1 7}$ & 12.5 & 7.5 & 1000 & $98 \pm 1$ & 0.253 & $-33 \pm 1$ \\
\hline $\mathbf{1 8}$ & 12.5 & 7.5 & 1500 & $96 \pm 2$ & 0.212 & $-34 \pm 2$ \\
\hline $\mathbf{1 9}$ & 15 & 2.5 & 500 & $125 \pm 1$ & 0.264 & $-32 \pm 2$ \\
\hline $\mathbf{2 0}$ & 15 & 2.5 & 1000 & $138 \pm 1$ & 0.257 & $-32 \pm 1$ \\
\hline $\mathbf{2 1}$ & 15 & 2.5 & 1500 & $180.4 \pm 0.3$ & 0.406 & $-28.0 \pm 0.3$ \\
\hline $\mathbf{2 2}$ & 15 & 5 & 500 & $138 \pm 2$ & 0.230 & $-34 \pm 2$ \\
\hline $\mathbf{2 3}$ & 15 & 5 & 1000 & $170 \pm 1$ & 0.331 & $-32 \pm 1$ \\
\hline $\mathbf{2 4}$ & 15 & 5 & 1500 & $102 \pm 1$ & 0.192 & $-32 \pm 1$ \\
\hline $\mathbf{2 5}$ & 15 & 7.5 & 1000 & $150 \pm 1$ & 0.352 & $-36 \pm 1$ \\
\hline $\mathbf{2 6}$ & 15 & 7.5 & 1500 & $92 \pm 2$ & 0.203 & $-38 \pm 2$ \\
\hline $\mathbf{2 7}$ & 15 & 7.5 & & & & $-34.5 \pm 0.2$ \\
\hline & & & 500 & & & \\
\hline
\end{tabular}

Figure 1B provides an overview of the data structure, identifying the groups of usNLCs sharing similar properties. Thus, it is possible to evaluate the usNLCs considering different formulations and process parameters. Five clusters were identified. Cluster 1 (red) represents usNLCs with a high $(>150 \mathrm{~nm}$ ) size and a PI > 0.250; cluster 2 (green) represents the high lipid content usNLC with high PS > $155 \mathrm{~nm}$; cluster 3 (blue) and 5 (purple) clusters show small usNLCs $(\leq 100-140 \mathrm{~nm}$ ) with a narrow distribution $<0.260$, but produced with different HPH processing times (5 and $7.5 \mathrm{~min}$ ); finally, cluster 4 (brown) displays the usNLCs with a high PS (>150 nm) but with a narrow distribution (0.160). The analysis shows that it is possible to increase the lipid content while obtaining a small sized formulation. During production, it is important to describe the relationship between HPH time and pressure. Figure 1B shows an easier interpretation for each cluster, confirming the similarity profile between formulations and the mean value to each corresponding variable in the respective cluster. 


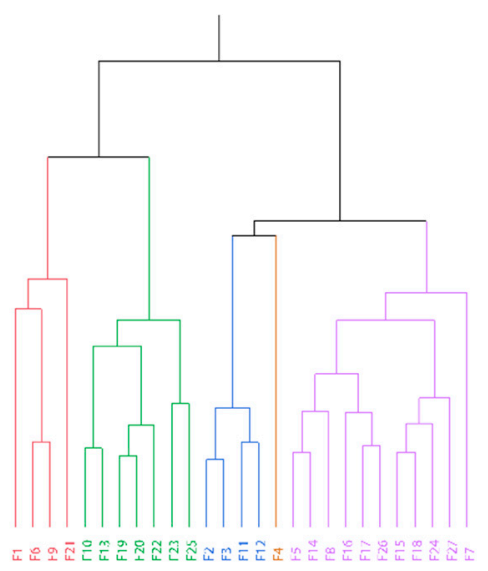

(A)

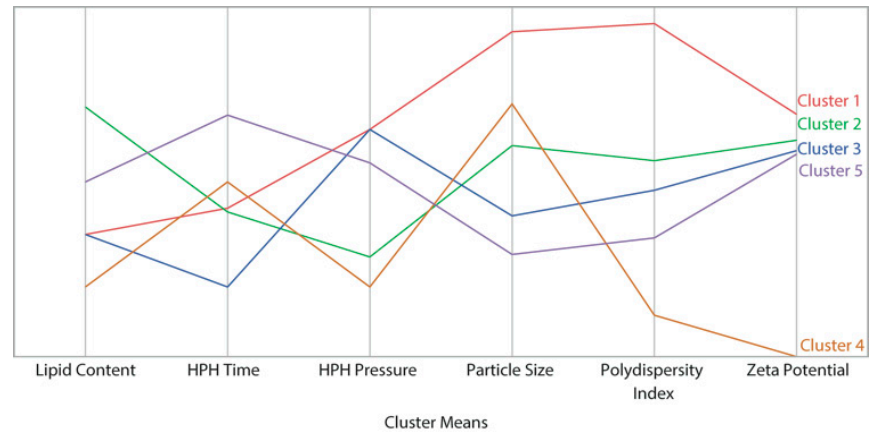

(B)

Figure 1. (A) Hierarchical cluster analysis (HCA) of experimental conditions taking into consideration their similarity in the expression of critical quality attributes (CQAs); and (B) cluster means representation.

Figure 2 presents the contribution of each independent and dependent variable, representing the 27 usNLC formulations. CPPs, as HPH pressure and HPH time, and CMAs, as lipid content, are explained by the first two principal components (PC), PC1, and PC2, which suggest the variables responsible for the discrimination of the formulations. The representation of data on the first two PCs is a simple and straightforward way to visualize and understand the relation between composition, process parameters and CQAs, discriminating the usNLCs formulations. The biplot allows visualizing the scores and loadings representing the coefficients of usNLCs, CPPs, CMAs and CQAs on the two principal components. Interpretation of the relative positioning of formulations on both the direction and length of the loadings is also important. PC1, the first component, retains information about the HPH time, while PC2, the second component, is associated to HPH pressure and lipid content. PS and PI contribute positively to PC1 and ZP to PC2. Therefore, HPH time opposes PS and PdI. This means a higher HPH time leads to a lower PS and narrow size distribution. HPH pressure and the lipid content are in the PC2 opposite direction, meaning that both parameters influence ZP. However, higher lipid content and higher HPH pressures lead to smaller ZP values. The effect of HPH pressure should be carefully inspected. High homogenization pressure might result in a decrease in the absolute value of ZP. Indeed, when the number of particles with high kinetic energy is increased, particle collision is favored, which may result in usNLC with lower stability. Despite all these trends, it should be noted that the $\mathrm{ZP}$ values remained above $130 \mid \mathrm{mV}$, ensuring formulation stability. 


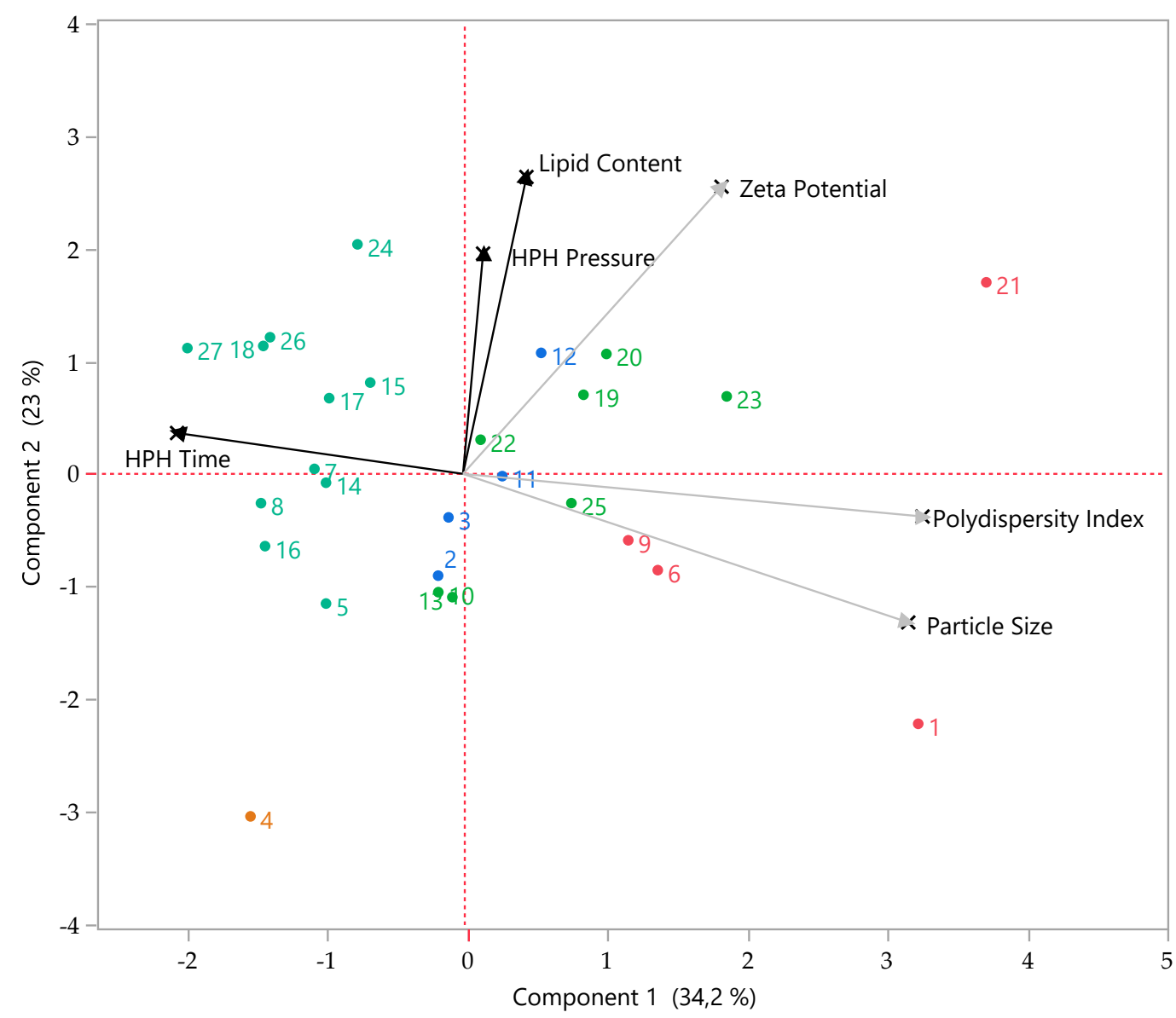

Figure 2. Principal component analysis (PCA) plotted along the first two axes, with the representation of the 27 usNLCs formulations and the 3 variables corresponding to usNLCs components and CQAs, on the first two components, recovering $57.2 \%$ of variance.

After understanding the similarities and patterns among formulations, a $3^{3}$ full factorial design was performed, now aiming at weighing the effect of the process parameters and the lipid content on the colloidal properties (PS, PI and ZP) of the usNLCs. Figure 3 exhibits the operable region for the optimized formulations. The model predicts that longer times in $\mathrm{HPH}(7.5 \mathrm{~min})$, high lipid content $(15 \% \mathrm{w} / \mathrm{w})$ ) and high pressures in $\mathrm{HPH}$ (ranging from 1000 and $1500 \mathrm{bar}$ ) lead to usNLCs with sizes below $100 \mathrm{~nm}$, narrow size distributions and $\mathrm{ZP}>|30| \mathrm{mV}$. Again, $\mathrm{ZP}$ is a parameter that does not seem to be affected by these variables (see Figure 3). 


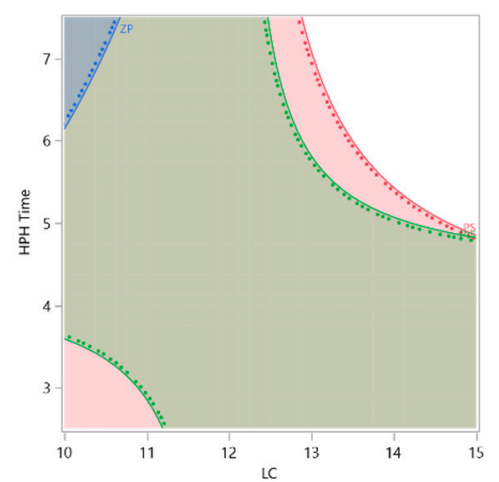

(A)

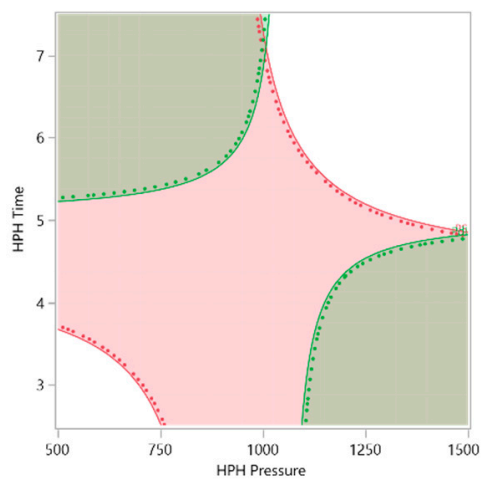

(B)

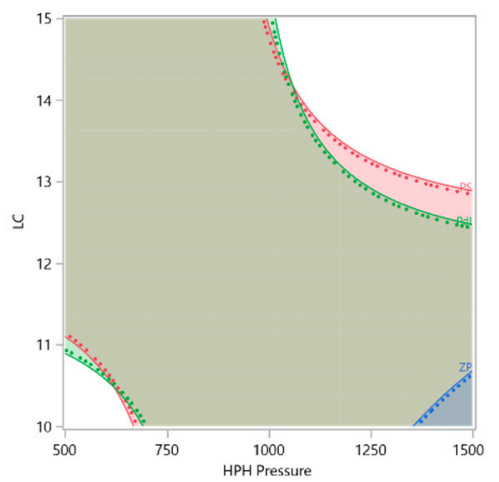

(C)

Figure 3. Particle size, polydispersity index and zeta potential contour plots for (A) HPH time vs. LC, (B) HPH time vs. HPH pressure and (C) LC vs. HPH pressure. The operable area is highlighted (white). Each missing factor was set to the maximum level, in order to optimize the CQAs.

\section{Discussion}

In a previous study $[9,10]$, it was possible to identify some key factors and understand which of them influence the mean particle size. These works addressed CMAs, such as lipid concentration, ratio between liquid and solid lipids, surfactant type, and concentration, and CPPs, such as Ultra-Turrax time, HPH pressure and batch volume. With the aim of increasing the lipid content, from 7.5 to $15 \% w / w$, and keeping the particle size below $100 \mathrm{~nm}$, it is now important to evaluate the influence of the usNLC production method and, consequently, the effect of increasing the lipid content on the usNLC properties. The QbD approach helped to understand the behavior between CPPs and CMAs in the hot$\mathrm{HPH}$ method and increase the lipid content compared to previous works [11,12]. It seems clear that usNLCs with higher lipid content yield larger particle sizes. However, with adjusted process parameters, longer HPH time and high pressures, it is possible to obtain particle sizes around $100 \mathrm{~nm}$ even with higher lipid content. A remarkable correlation was found between the tested parameters and the mean particle diameter. A high HPH pressure along with extended HPH time results in smaller usNLC for the tested set-up. Furthermore, narrow PIs are associated with high pressures, between 1000 and 1500 bar, irrespective of time. Attempting a translation to cancer drug delivery, usNLC with these properties are expected to hold an enhanced permeability and retention (EPR) effect with a preferential accumulation in the tumor tissues. Moreover, particle size stands as a critical parameter for intravenous delivery and permeation through biological barriers. However, future studies must be carried out to explore the therapeutic potential of usNLCs in cancer.

\section{Conclusions}

The QbD-based optimization method was successfully implemented as a surrogate to the conventional trial-and-error approach. Multivariate analyses were applied to infer about the critical parameters influencing the physicochemical characteristics, such as PS, $\mathrm{PI}$, and ZP, of the usNLC formulated by the hot-HPH method. Understanding the parameters that should be used in the hot-HPH method for usNLC represents a useful approach for subsequent optimization and characterization steps. The optimal formulation was obtained in terms of size and polydispersity with a high lipid content $(15 \% w / w)$, a long time in HPH (7.5 min), and an intermediate HPH pressure (1000 bar). In conclusion, this work provides crucial information on the process parameters and critical materials attributes for usNLC production targeting cancer therapy. 
Author Contributions: M.M. performed the experiments and analyzed the data; J.S., A.P. and C.V. contributed reagents/materials/analysis tools; M.M., J.B., J.S., A.P. and C.V. contributed to the writing and editing of the paper. All authors have read and agreed to the published version of the manuscript.

Institutional Review Board Statement: Not applicable.

Informed Consent Statement: Not applicable.

Data Availability Statement: Data sharing not applicable. No new data were created or analyzed in this study. Data sharing is not applicable to this article.

Acknowledgments: The Coimbra Chemistry Centre is also supported by FCT through the Project UID/QUI/00313/2020. Maria Mendes and João Basso and acknowledge the Ph.D. research Grants SFRH/BD/133996/2017 and SFRH/BD/149138/2019, respectively, assigned by FCT.

Conflicts of Interest: The authors declare no conflict of interest.

$\begin{array}{ll}\text { Abbreviations } \\ \text { usNLC } & \text { Ultra-small nanostructured lipid carriers } \\ \text { NPs } & \text { Nanoparticles } \\ \text { QbD } & \text { Quality by design } \\ \mathrm{HPH} & \text { High-pressure homogenization } \\ \mathrm{CPP} & \text { Critical process parameters } \\ \text { CMAs } & \text { Critical material attributes } \\ \text { CQAs } & \text { Critical quality attributes } \\ \text { LC } & \text { Lipid content } \\ \text { PS } & \text { Particle size } \\ \text { PI } & \text { Polydispersity index } \\ \text { ZP } & \text { Zeta potential } \\ \text { F } & \text { Formulation }\end{array}$

\section{References}

1. Patel, G.M.; Shelat, P.K.; Lalwani, A.N. QbD based development of proliposome of lopinavir for improved oral bioavailability. Eur. J. Pharm. Sci. 2017, 108, 50-61, doi:10.1016/j.ejps.2016.08.057.

2. Politis, S.N.; Colombo, P.; Colombo, G.; Rekkas, D.M. Design of experiments (DoE) in pharmaceutical development. Drug Dev. Ind. Pharm. 2017, 43, 889-901, doi:10.1080/03639045.2017.1291672.

3. Cunha, S.; Costa, C.P.; Moreira, J.N.; Lobo, J.M.S.; Silva, A.C. Using the quality by design (QbD) approach to optimize formulations of lipid nanoparticles and nanoemulsions: A review. Nanomed. Nanotechnol. Biol. Med. 2020, 28, 102206, doi:10.1016/ j.nano.2020.102206.

4. Tamjidi, F.; Shahedi, M.; Varshosaz, J.; Nasirpour, A. Nanostructured lipid carriers (NLC): A potential delivery system for bioactive food molecules. Innov. Food Sci. Emerg. Technol. 2013, 19, 29-43.

5. Monteagudo, S.M.; Yan, B.; Balasubramaniam, V.M. Engineering Process Characterization of High-Pressure HomogenizationFrom Laboratory to Industrial Scale. Food Eng. Rev. 2016, 9, 143-169, doi:10.1007/s12393-016-9151-5.

6. Dingler, A.; Gohla, S. Production of solid lipid nanoparticles (SLN): scaling up feasibilities. J. Microencapsul. 2002, 19, 11-16, doi:10.1080/02652040010018056.

7. Gupta SNRCN. Advances in Nanomedicine for the Delivery of Therapeutic Nucleic Acids; Elsevier: Amsterdam, The Netherlands, 2017.

8. Thassu, D.; Deleers, M.; Pathak, Y.V.; Pathak, Y. Nanoparticulate Drug Delivery Systems [Internet]. Taylor \& Francis. (Drugs and the Pharmaceutical Sciences). 2007. Available from: https://books.google.pt/books?id=B-hsAAAAMAAJ (accessed on 30 November 2020).

9. Barone, A.; Mendes, M.; Cabral, C.; Mare, R.; Paolino, D.; Vitorino, C. Hybrid Nanostructured Films for Topical Administration of Simvastatin as Coadjuvant Treatment of Melanoma. J. Pharm. Sci. 2019, 108, 3396-3407, doi:10.1016/j.xphs.2019.06.002.

10. Mendes, M.; Soares, H.; Arnaut, L.; Sousa, J.; Pais, A.; Vitorino, C. Can lipid nanoparticles improve intestinal absorption? Int. J. Pharm. 2016, 515, 69-83, doi:10.1016/j.ijpharm.2016.09.065. 
11. Mendes, M.; Basso, J.; Silva, J.; Cova, T.; Sousa, J.; Pais, A.; Vitorino, C. Biomimeting ultra-small lipid nanoconstructs for glioblastoma treatment: A computationally guided experimental approach. Int. J. Pharm. 2020, 587, 119661, doi:10.1016/j.ijpharm.2020.119661.

12. Basso, J.; Mendes, M.; Silva, J.; Sereno, J.; Cova, T.; Oliveira, R.; Fortuna, A.; Castelo-Branco, M.; Falcão, A.; Sousa, J.; et al. Peptide-lipid nanoconstructs act site-specifically towards glioblastoma growth impairment. Eur. J. Pharm. Biopharm. 2020, 155, 177-189, doi:10.1016/j.ejpb.2020.08.015. 